\title{
SalA Attenuates Ischemia/Reperfusion- Induced Endothelial Barrier Dysfunction via Down-Regulation of VLDL Receptor Expression
}

\author{
Dan Yang ${ }^{\mathrm{a}}$ Peng Zhang ${ }^{\mathrm{a}}$ Tingfeng Wang ${ }^{\mathrm{b}}$ Lili Gao $^{\mathrm{a}}$ Zhengdong Qiao \\ Yongjun Lianga Bo Yu ${ }^{\mathrm{c}}$ \\ ${ }^{a}$ Center for Medical Innovation and Research, bepartment of General Surgery, \\ 'Department of Vascular Surgery, Shanghai Pudong Hospital, Fudan University, Shanghai
}

\section{Key Words}

Salvianolic acid A $\bullet$ Ischemia/reperfusion $•$ VLDL receptor $•$ Endothelial barrier dysfunction

\begin{abstract}
Background: Salvianolic acid A (SalA) has been shown to confer robust protection against endothelial injury. VLDL receptor is expressed at high levels on the endothelial surface, however its biological effect on endothelial cells has not yet been completely elucidated. Here, we investigated molecular effects of SalA on endothelial VLDL expression and barrier dysfunction under conditions of ischemia/reperfusion (IS/RP). Methods: Human umbilical vein endothelial cells (HUVECs) treated with SalA were subjected to IS/RP stimulation. Endothelial permeability, ZO-1 distribution, actin cytoskeleton reorganization, and intracellular reactive oxygen species (ROS) generation were examined. The mRNA levels were tested by real-time RT-PCR and the protein levels were determined by immunoblot analysis. Results: Pretreatment of HUVECs with SalA markedly attenuated IS/RP-induced endothelial hyperpermeability, discontinuous ZO-1 staining, actin stress fiber formation, and intracellular ROS generation. IS/RP activated p38 MAPK signaling and enhanced VLDL receptor expression, and inactivation of p38 MAPK abolished increase of VLDL receptor expression. Furthermore, siRNA experiments showed that VLDL receptor was a crucial mediator of endothelial barrier dysfunction and intracellular ROS generation induced by IS/RP. Importantly, SalA effectively suppressed IS/RP-induced activation of p38 MAPK signaling and increase of VLDL receptor expression. Conclusion: These results for the first time demonstrated that SalA protected against IS/RP-induced endothelial barrier dysfunction through suppression of VLDL receptor expression.
\end{abstract}

Copyright (c) 2014 S. Karger AG, Basel

Bo Yu, M.D.
Department of Vascular Surgery, Pudong Hospital, Fudan University,

\#2800 Gongwei Roud, Huinan Town, Pudong New Area, Shanghai 201399 (P.R. China)

Tel. +86-21-6803-6516, Fax +86-21-6803-6516, E-Mail dyang0226@163.com 
Yang et al.: SalA Protects IS/RP-Induced Endothelial Barrier Dysfunction

\section{Introduction}

The vascular endothelium consisting of a single cell layer lining all vessels, acts as a physiological barrier and plays an important role in maintaining the vascular homeostasis. Damage to the endothelial barrier results in pathological vascular hyperpermeability, which is commonly attributed to the reorganization of actin cytoskeleton, followed by contraction of cells and formation of paracellular gaps [1]. Multiple pathological events and proinflammatory cytokines, such as the ischemic/reperfusion (IS/RP) injury, transforming growth factorbeta (TGF- $\beta$ ), and tumor necrosis factor-alpha (TNF- $\alpha$ ) can trigger downstream signals that initiate reorganization of the actin cytoskeleton and thus the endothelial hyperpermeability and barrier dysfunction [2,3].

The very-low-density-lipoprotein (VLDL) receptor is a transmembrane lipoprotein receptor of the low-density-lipoprotein (LDL) receptor family. VLDL receptor is found throughout the body, with particularly high expression in fatty acid tissues due to their high level of triglycerides, VLDL receptor's primary ligand. These tissues include those of the heart, skeletal muscle, and adipose layer. In addition, the receptor is also found in endothelial cells $[4,5]$. The biological function of VLDL receptor is associated with lipoprotein uptake, endocytosis, neuronal migration and so on. Recently, some study indicates a role of VLDL receptor in the endothelial pathology. It was suggested that VLDL receptor mediated activation of retinal vascular endothelial cells and promotion of angiogenesis $[6,7]$. However, no further study was provided to elucidate the effects of VLDL receptor on the other endothelial pathological events, such as barrier dysfunction.

S. miltiorrhiza, commonly named "Danshen" in China, is a valuable medicinal plant [8], and can be used to alleviate some significant symptoms of cardiovascular disease. It is also an important ingredient of compound danshen dripping pills which have been passed phase II human clinical trial of U.S. Food and Drug Administration (FDA). Salvianolic acid A (SalA), is one of the main active, water-soluble components in S. miltiorrhiza. Previous studies have indicated the beneficial effects of SalA on preventing oxidative stress, platelet aggregation, ischemia, and hepatocirrhosis [9]. In the present study, we, for the first time, provide evidence for the protective effect of SalA on IS/RP-induced endothelial barrier dysfunction through down-regulation of VLDL receptor expression in human umbilical endothelial cells (HUVECs).

\section{Materials and Methods}

\section{Chemicals and reagents}

Anti-VLDL receptor (VLDL-R) antibody was obtained form R\&R Systems, and Anti-GAPDH and HRPconjugated secondary antibodies were from Cell Signaling Technology. SB203580 was obtained from Calbiochem. ROS detection kit was purchased from Beyotime Company (Jiangsu, China). All other chemicals were from Sigma.

\section{Cell culture}

Human umbilical endothelial cells (HUVECs) (Sciencell) were cultured in endothelial cell medium (ECM) supplemented with endothelial cell growth supplement (ECGS), 5\% fetal bovine serum (FBS), and penicillin/streptomycin (P/S) solution (Sciencell) in an incubator with $37^{\circ} \mathrm{C}, 5 \% \mathrm{CO}_{2}$. For experiments, cells of passage no. 4-6 were grown until confluence. In some experiments, HUVECs were pretreated with SalA or SB203580 for $30 \mathrm{~min}$ and then subjected to the stimulated IS/RP protocol.

\section{Stimulated IS/RP experimental protocol}

HUVECs were subjected to oxygen-glucose deprivation (OGD) injury [10,11]. The OGD injury was initiated by placing cells in a tri-gas incubator with an atmosphere of $37^{\circ} \mathrm{C}, 5 \% \mathrm{CO}_{2}, 94 \% \mathrm{~N} 2$, and $1 \% \mathrm{O}_{2}$, and in the presence of OGD media for $5 \mathrm{~h}$ (IS). After that, the exposure medium was replaced with oxygenated $\mathrm{ECM}$, and the cells were placed in an incubator at $37^{\circ} \mathrm{C}, 5 \% \mathrm{CO}_{2}(\mathrm{RP})$, to mimic the reperfusion conditions at different time points as indicated. 
Yang et al.: SalA Protects IS/RP-Induced Endothelial Barrier Dysfunction

Permeability assay

HUVECs were cultured in the transwell plates $(0.4 \mathrm{~mm}$, Costar). Cells were pretreated with SalA for $30 \mathrm{~min}$ and subjected to IS $5 \mathrm{~h}$ and RP $6 \mathrm{~h}$. Then, fluorescein-isothiocyanate-labelled bovine serum albumin (BSA) (FITC-BSA, $10 \mathrm{mg} / \mathrm{mL}$, Sigma) was given to the cells. Cell culture medium from the lower compartment was removed after $60 \mathrm{~min}$. FITC-BSA was quantified in a fluorescence spectrofluorophotometer. Bovine serum albumin flux was valued as a ratio between fluorescence intensities in the lower compartment (60 $\min$ ) and the upper compartment (0 min) [12].

\section{Immunofluorescence staining of $\mathrm{ZO}-1$ protein}

HUVECs were fixed at room temperature in PBS containing 2\% paraformaldehyde for 10 min. After washes with PBS, cells were permeabilized at room temperature for 5 min with $0.1 \%$ Triton X-100. Cells were also blocked with 2\% BSA in PBS at room temperature for $30 \mathrm{~min}$ and washed with PBS. After that, cells were incubated with primary antibody to ZO-1 $(0.5 \mathrm{ug} / \mathrm{ml})$ (BD Biosciences) for $48 \mathrm{~h}$ at $4^{\circ} \mathrm{C}$. After three time washes with PBS, cells were incubated with fluorescent conjugated secondary antibodies (BD Biosciences). After that, nuclei were counterstained with the DNA-binding dye 2-(4-amidinophenyl)-6indolecarbamidinedihydrochloride (DAPI). Stained HUVECs were observed under a laser confocal scanning microscope (TCS SP2; Leica).

\section{Actin cytoskeleton staining}

Actin cytoskeleton was stained using the Rhodamine-Phalloidin Based F-Actin Visualization Biochem Kit (Cytoskeleton) according to the manufacture's instructions. Briefly, HUVECs were cultured and fixed with paraformaldehyde and permeabilized for 5 min with $0.1 \%$ Triton X-100. Cells were then incubated with a $1 \%$ solution of BSA for $30 \mathrm{~min}$ in room temperature, and stained with rhodamine-phalloidin [12]. After that, nuclei were counterstained with the DAPI. Stained HUVECs were visualized using a laser confocal scanning microscope (TCS SP2; Leica).

\section{Image analysis of gap formation}

To detect the formation of paracellular gaps, images of rhodamine-stained endothelial cell monolayers were processed with MetaVue 4.6 software (Universal Imaging, Downington, PA, USA) as described previously [12]. Briefly, paracellular gaps were manually marked out, and images were differentially segmented between gaps (black) and cells (highest gray value) based on image gray scale levels. The gap formation was expressed as a ratio of the gap area to the area of the whole image. The values were statistically processed using Sigma Plot 7.1 (SPSS Science, Chicago, IL, USA) software.

\section{Determination of ROS generation}

The intracellular ROS levels were determined by measuring the oxidative conversion of cell permeable 2'7'-dichlorofluorescein diacetate (DCFH-DA) to fluorescent dichlorofluorescein (DCF) in a fluorospectrophotometer (F4000, Japan). Briefly, cells in 6-well culture dishes were pretreated with SalA for 30 min and subjected to IS $5 \mathrm{~h}$ and RP $6 \mathrm{~h}$, and then were washed with D-Hank's and incubated with DCFH-DA at $37^{\circ} \mathrm{C}$ for $20 \mathrm{~min}$. Then DCF fluorescence distribution of cells was analyzed by a fluorospectro-photometer at an excitation wavelength of $488 \mathrm{~nm}$ and at an emission wavelength of $525 \mathrm{~nm}$.

\section{SiRNA transfection}

HUVECs were transfected with $50 \mathrm{nmol} / \mathrm{L}$ VLDL receptor (VLDL-R) siRNA or nontargeting siRNA obtained from Dharmacon as described previously [12]. The nontargeting siRNA was used as transfection control for non-sequence-specific effects of the transfected siRNAs.

\section{Quantitative real-time RT-PCR analysis}

Total RNA was purified from HUVECs with Trizol (Invitrogen). Realtime PCR was performed using Bio-RAD iQ5 Multicolor Real-Time PCR Detection System with SYBR Green as fluorescent and ROX (Takara) as reference dyes as described previously [13]. The specific primers used were as follows: human VLDL receptor: 5'-GTGGAAAATGTGATGGGGATG-3' (forward), 5'-CCATTGTTGCACACGAAGTC-3' (reverse); Human GAPDH: 5'-GACCCCTTCATTGACCTC-3' (forward), 5'-GCTAAGCAGTTGGTGGTG-3' (reverse). 
Fig. 1. SalA inhibited IS/RPinduced endothelial barrier dysfunction. HUVECs were pretreated with SalA for 30 min in various concentrations, and then subjected to IS 5h/RP 6h treatment. (A) Box plot showed endothelial permeability. $(n=4$ per group). ${ }^{*} \mathrm{P}<0.05$ vs. untreated control cells; \#P<0.05 vs. non-SalA-treated IS/RP cells. The line within the box plot corresponds to the median value. (B) Z0-1 was labelled using anti-ZO-1 antibody. Actin cytoskeleton was stained with rhodamine-phalloidin. A representative field was shown for each condition. Paracellular gaps were marked by arrows (scale bars: $25 \mathrm{~mm}$ ). (C) Box plot showed gap area quantitated by morphometric analysis of rhodamine-phalloidin-stained HUVECs ( $n=4$ per group). ${ }^{*} \mathrm{P}<0.05$ vs. untreated control cells; \#P<0.05 vs. nonSalA-treated IS/RP cells. The line within the box plot corresponds to the median value.

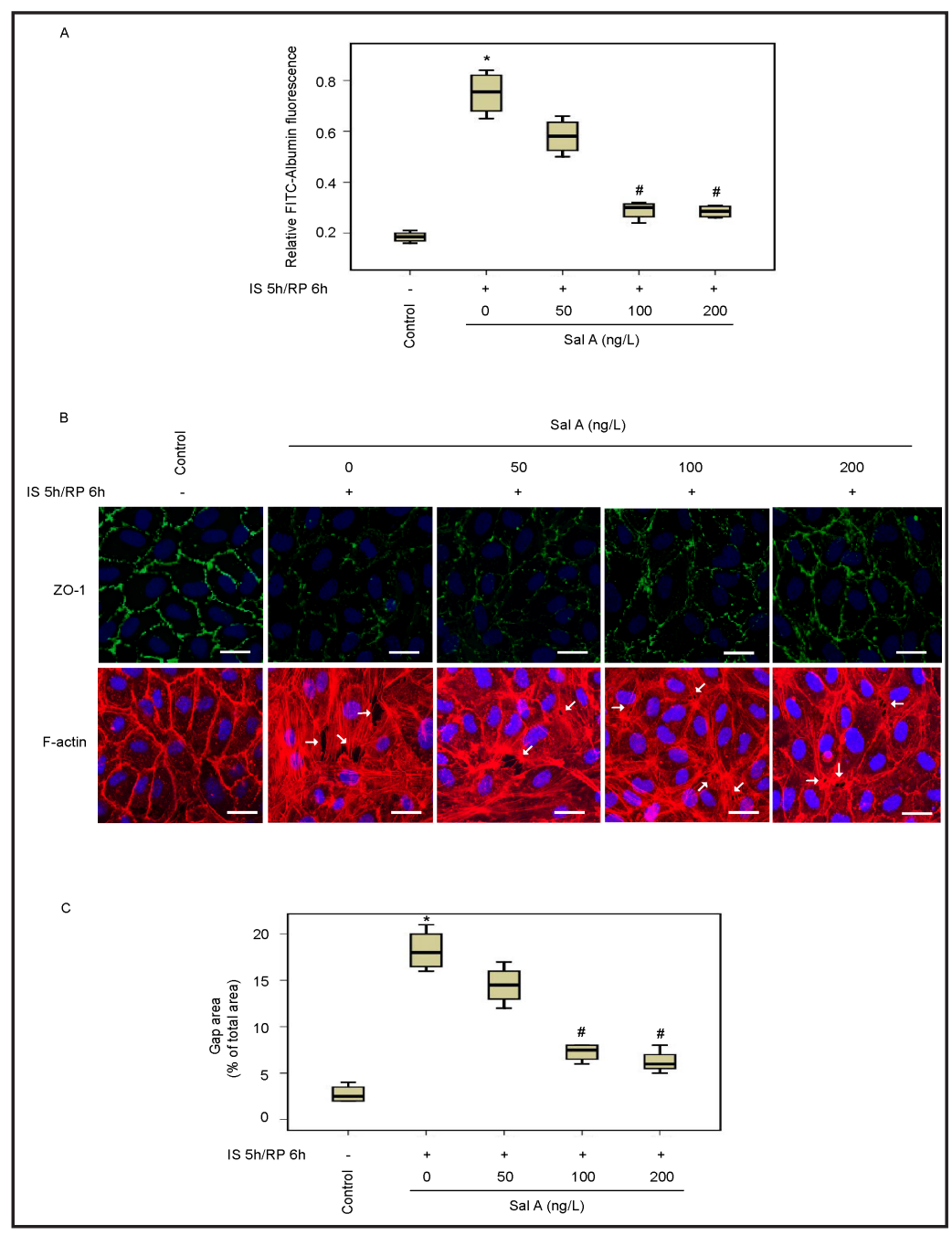

Western blot analysis

Immunoblotting was performed according to the previous protocols [11]. Total proteins were extracted from HUVECs using cell lysis buffer. Protein (40-60 ug) from each sample were separated by SDS-PAGE, transferred to nitrocellulose membranes, and probed with primary antibodies and then with horseradish peroxidase-conjugated secondary antibodies. The enhanced chemiluminescence signal was quantified using a densitometry program (Gel-pro 4.5 Analyzer, Media Cybernetics). To quantify the protein signal, we subtracted background, normalized the value to GAPDH. As for the phospho-specific protein, we normalized the signal to the amount of total target protein and GAPDH.

Statistical analysis

Each experiment was performed at least in triplicate. For comparisons between two groups the MannWhitney U test for non-parametric data was performed. The data were considered to be significant at $P<0.05$. SPSS software was used for data analysis.

\section{Results}

SalA inhibited IS/RP-induced endothelial hyperpermeability

We firstly investigated the biological role of SalA in IS/RP-induced endothelial dysfunctions. According to our previous research, the SalA concentrations of 50, 100 and 
Fig. 2. SalA attenuated IS/RP-induced intracellular ROS generation. HUVECs were pretreated with SalA for $30 \mathrm{~min}$ in various concentrations, and then subjected to IS 5h/RP $6 \mathrm{~h}$ treatment. Box plot showed intracellular ROS level in each group. ( $n=4$ per group). ${ }^{*} P<0.05$ vs. untreated control cells; ${ }^{\#} P<0.05$ vs. non-SalA-treated IS/RP cells. The line within the box plot corresponds to the median value.
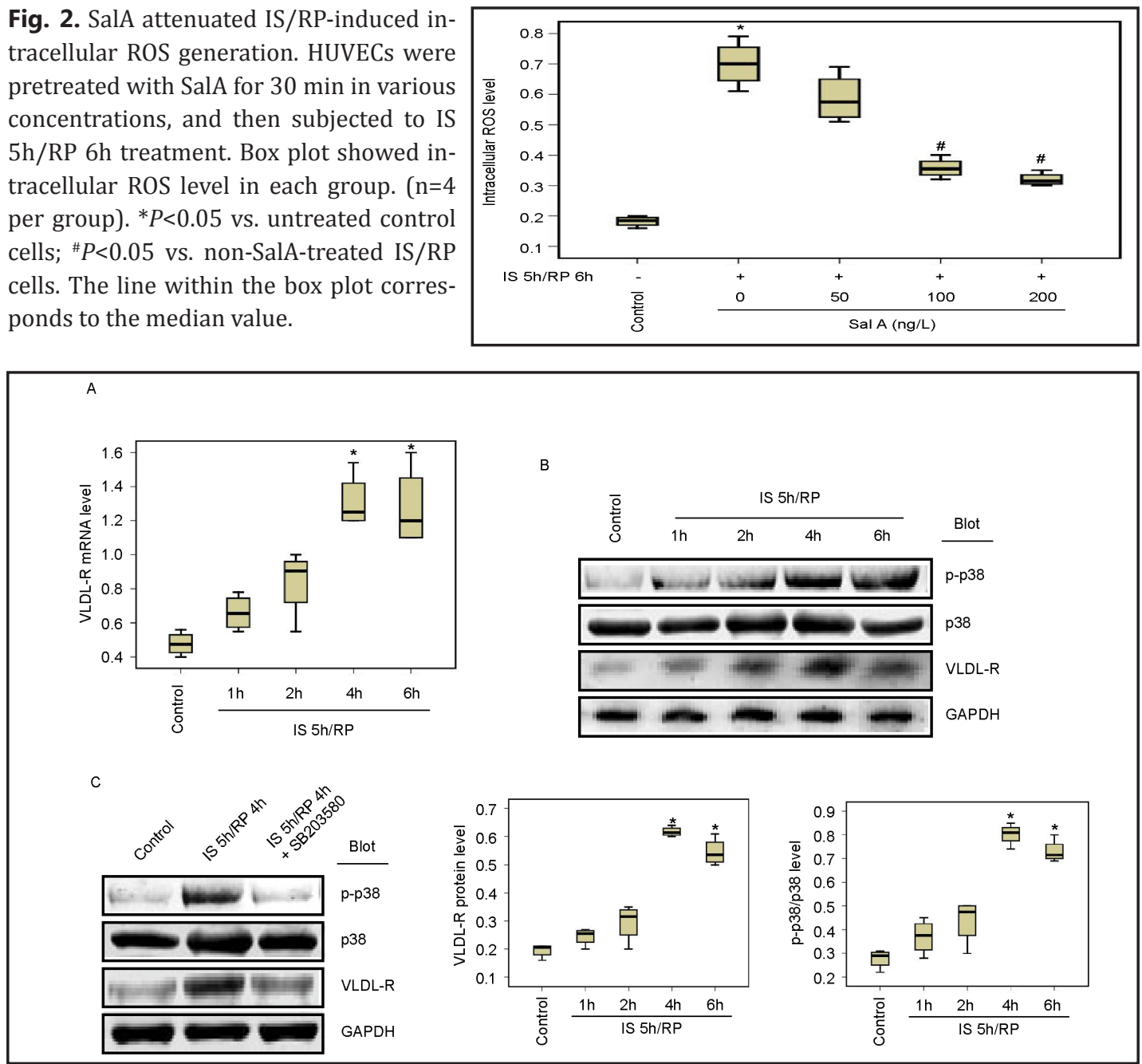

Fig. 3. IS/RP induced expression of VLDL receptor. (A) HUVECs were subjected to IS for 5h and RP for indicated time points. Box plot showed the VLDL receptor mRNA levels in each group detected by quantitative real-time RT-PCR ( $=4$ per group). ${ }^{*} \mathrm{P}<0.05$ vs. untreated control cells. The line within the box plot corresponds to the median value. (B) HUVECs were treated as in (A). The protein levels of p-p38, p38, VLDL receptor and GAPDH were detected by immunoblot analysis using indicated antibodies. A representative blot (top) was shown. Box plot showed the expression levels of p-p38 and VLDL receptor (bottom) ( $\mathrm{n}=4$ per group). ${ }^{*} P<0.05$ vs. untreated control cells. The line within the box plot corresponds to the median value. (C) HUVECs were pretreated with SB203580 (10 mmol/L) for 30min prior to IS/RP treatment. The protein levels of p-p38, p38, VLDL receptor, and GAPDH were detected by immunoblot analysis using indicated antibodies and a representative blot was shown $(n=4)$.

200ng/L were protective in IS/RP-induced endothelial injury [13], thus we treated cells with SalA at concentrations of 50,100 and 200ng/L in the present study. As shown in Figure 1A, treatment of cells with IS/RP resulted in higher endothelial perpermeability than control cells, and pretreatment of cells with SalA at the concentration of 100 or $200 \mathrm{ng} / \mathrm{L}$ markedly attenuated IS/RP-induced endothelial hyperpermeability. Interestingly, in HUVECs under control condition, cells exhibited continuous membranous staining of ZO-1, a cytoplasmic plaque protein of tight junctions (Fig. 1B).After IS/RP, cells showed discontinuous distribution and decreased intensity of Z0-1 staining, which was largely restored by pretreatment with SalA at the concentration of 100 or 200ng/L (Fig. 2B). Further, Figure 1B showed that IS/RP resulted in a significant formation of actin stress fibers, and SalA markedly inhibited IS/RPinduced actin cytoskeleton alterations at the concentration of 100 or 200ng/L. In addition, 


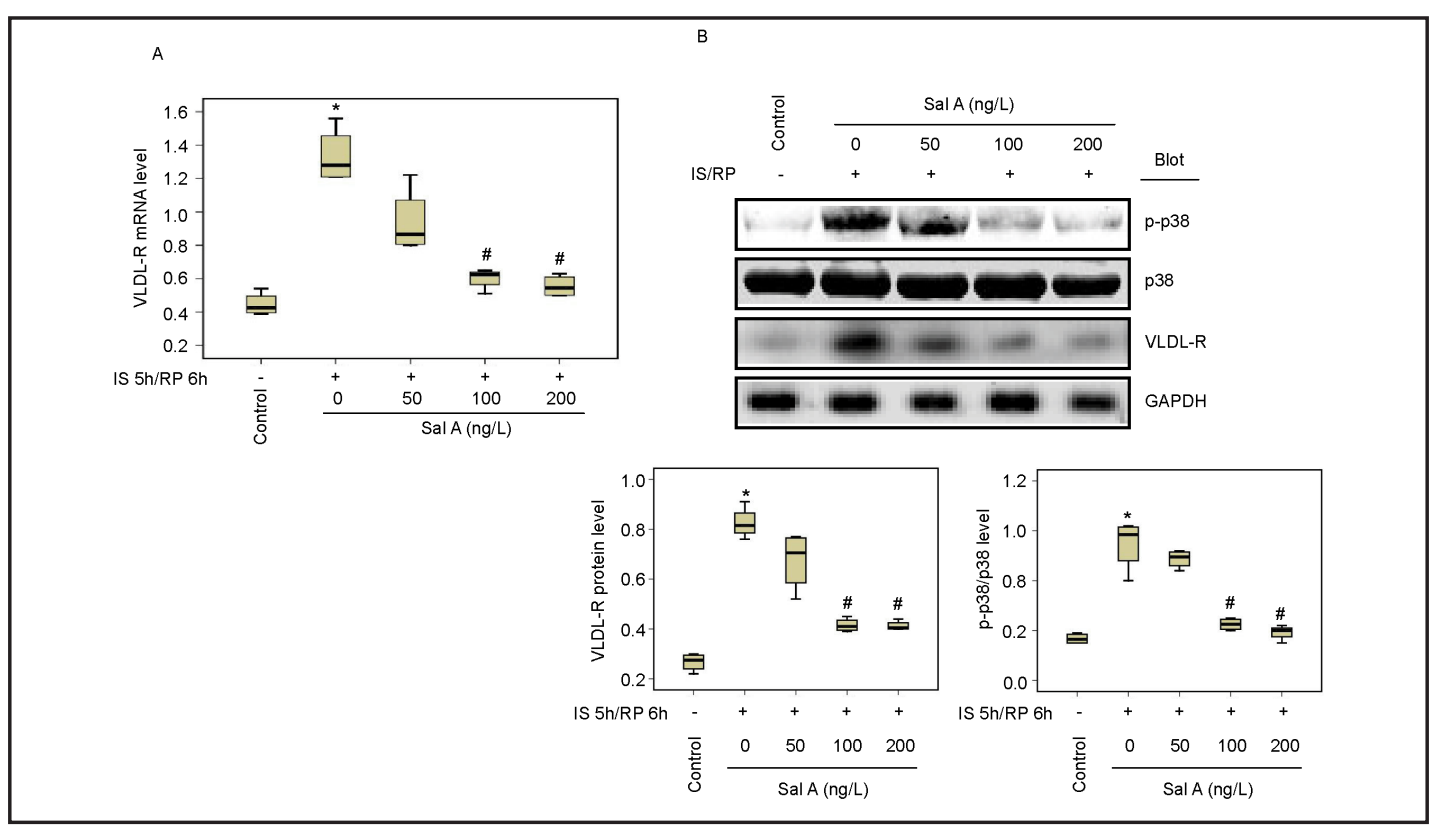

Fig. 4. SalA inhibited IS/RP-enhanced expression of VLDL receptor. (A) HUVECs were pretreated with SalA for $30 \mathrm{~min}$ in various concentrations, and then subjected to IS 5h/RP $6 \mathrm{~h}$ treatment. Box plot showed the VLDL receptor mRNA levels in each group detected by quantitative real-time RT-PCR ( $\mathrm{n}=4$ per group). ${ }^{*} P<0.05$ vs. untreated control cells; ${ }^{\#} P<0.05$ vs. non-SalA-treated IS/RP cells. The line within the box plot corresponds to the median value. (B) HUVECs were treated as in (A). The protein levels of p-p38, p38, VLDL receptor, and GAPDH were detected by immunoblot analysis using indicated antibodies. A representative blot (top) was shown. Box plot showed the expression levels of p-p38 and VLDL receptor (bottom) in each group ( $\mathrm{n}=4$ per group). ${ }^{*} P<0.05$ vs. untreated control cells; ${ }^{\#} P<0.05$ vs. non-SalA-treated IS/RP cells. The line within the box plot corresponds to the median value.

the paracellular gap area was also measured. IS/RP lead to increased paracellular gap area, SalA markedly ameliorated enlargement of intracellular space at the concentration of 100 or 200ng/L (Fig. 2B).

\section{SalA attenuated IS/RP-induced endothelial ROS generation}

The intracellular ROS generation is a crucial mediator to trigger cytoskeleton reorganization and endothelial hyperpermeability $[14,15]$. Thus, we investigated whether SalA function as an effective anti-oxidant in endothelial cells after IS/RP. Compared with control cells, IS/RP significantly increased the intracellular level of ROS (Fig. 2). Pretreatment of cells with SalA (100 or 200ng/L) effectively inhibited the elevated intracellular concentration of ROS induced by IS/RP. However, low concentration of 50ng/L had no significant effect on the intracellular level of ROS (Fig. 2).

\section{IS/RP induced expression of VLDL receptor}

In our preliminary microarray test, we found that mRNA expression of VLDL receptor was induced by IS/RP and inhibited by SalA pretreatment (data not shown). Thus, we firstly tested expression of VLDL receptor under condition of IS/RP. As shown in Figure 3A and B, the VLDL receptor expression was increased by IS/RP treatment and peaked at reperfusion for 4 hour, followed by a decline at reperfusion for 6 hour. It is reported that activation of p38 MAPK signaling plays an essential role in IS/RP-induced endothelial injury $[16,17]$. In our results, IS/RP triggered the activation of p38 MAPK signaling significantly at the time points of 4 and 6 hour (Fig. 3B). Interestingly, inactivation of p38 MAPK by SB203580 largely abolished the enhanced expression of VLDL receptor under condition of IS/RP (Fig. 3C). 
Fig. 5. Inhibition of VLDL receptor expression inhibited endothelial barrier dysfunction. HUVECs were non-transfected (-) or transfected with VLDL receptor siRNA (50 nmol/L, siVLDLR) or nontargeting siRNA ( $50 \mathrm{nmol} / \mathrm{L}$, siNT). After $48 \mathrm{~h}$, cells were non-pretreated or pretreated with $100 \mathrm{ng} / \mathrm{L}$ SalA. Then cells were subjected to the treatment of IS 5h/RP $6 \mathrm{~h}$ treatment. (A) The protein levels of VLDL receptor and GAPDH were detected by immunoblot analysis using indicated antibodies. A representative blot was shown ( $n=3)$. (B) Box plot showed the endothelial permeability in each group. ( $n=4$ per group). ${ }^{*} P<0.05$ vs. untreated control cells; ${ }^{\#} P<0.05$ vs. non-transfected IS/RP cells. The line within the box plot corresponds to the median value. (C) Box plot showed the gap area quantitated by morphometric analysis of rhodamine-phalloidin-stained HUVEC ( $\mathrm{n}=4$ per group). ${ }^{*} P<0.05$ vs. untreated control cells; ${ }^{\#} P<0.05$ vs. non-transfected IS/RP cells. The line within the box plot corresponds to the median value.

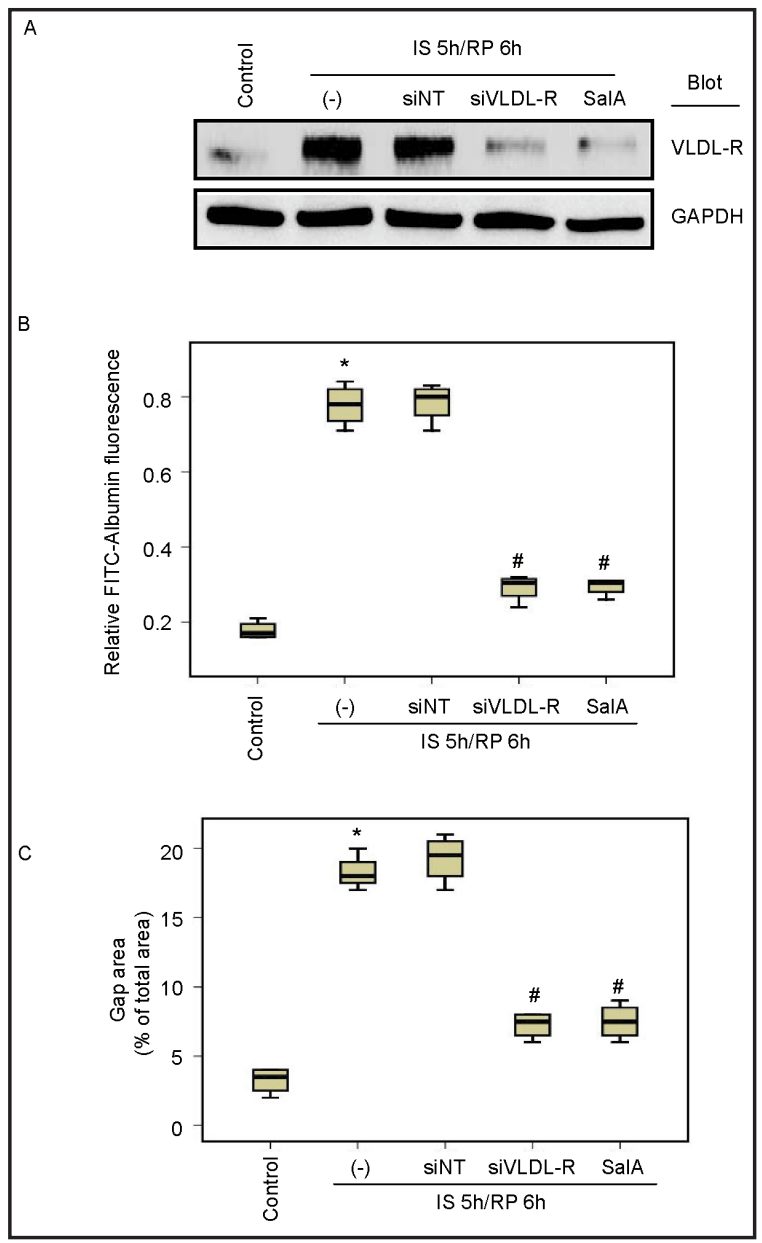

SalA inhibited IS/RP-enhanced expression of VLDL receptor

Next, we tested whether SalA altered the VLDL receptor expression. As shown in Figure $4 \mathrm{~A}$ and $\mathrm{B}, \mathrm{IS} / \mathrm{RP}$ resulted in increase of VLDL receptor mRNA and protein levels, which was sufficiently abolished by SalA treatment at the concentration of 100 or $200 \mathrm{ng} / \mathrm{L}$. Importantly, corresponding to the SalA-altered VLDL receptor expression, IS/RP-induced activation of p38 MAPK signaling was also suppressed by SalA treatment markedly (Fig. 4B).

Suppression of VLDL receptor expression inhibited endothelial hyperpermeability

To investigate the role of endogenous VLDL receptor expression in IS/RP-induced endothelial dysfunction, we used the siRNA technique to suppress VLDL receptor expression in HUVECs. We first optimized the conditions for cell transfection and tested VLDL receptor expression level. A significant reduction of VLDL receptor expression was observed in cells transfected with VLDL receptor siRNA compared with non-targeting siRNA (Fig. 5A). Furthermore, VLDL receptor siRNA markedly abolished the IS/RP-induced hyperpermeability and paracellular gap formation, whereas non-targeting siRNA had no effect (Fig. 5B, C). Additionally, the protective effect of SalA in IS/RP-induced endothelial barrier dysfunction was similar with the endothelial response triggered by VLDL receptor siRNA treatment (Fig. 5B, C).

Suppression of VLDL receptor expression attenuated intracellular ROS generation

We further detected the role of VLDL receptor in IS/RP-induced ROS generation. As shown in Figure 6, transfection of cells with VLDL receptor siRNA markedly abolished the 
Fig. 6. Inhibition of VLDL receptor expression attenuated intracellular ROS generation. HUVECs were nontransfected (-) or transfected with VLDL receptor siRNA $(50 \mathrm{nmol} / \mathrm{L}$, siVLDL-R) or nontargeting siRNA (50 $\mathrm{nmol} / \mathrm{L}$, siNT). After $48 \mathrm{~h}$, cells were non-pretreated or pretreated with $100 \mathrm{ng} / \mathrm{L}$ SalA. Then cells were subjected to the treatment of IS 5h/RP $6 \mathrm{~h}$ treatment. Box plot showed intracellular ROS level in each group ( $n=4$ per group). ${ }^{*} P<0.05$ vs. untreated control cells; ${ }^{\#} P<0.05$ vs. non-transfected IS/ RP cells. The line within the box plot corresponds to the median value.

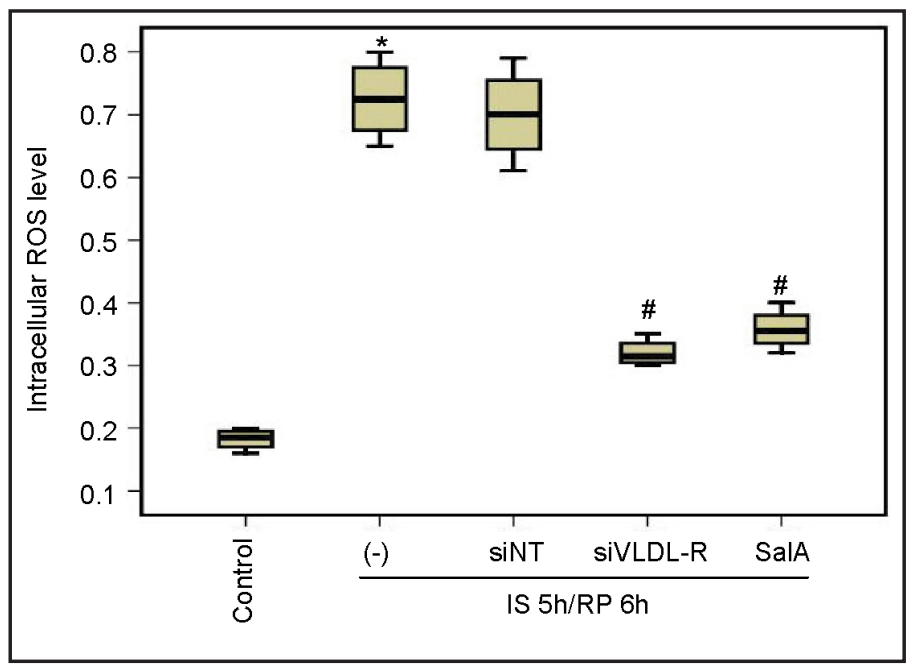

IS/RP-enhanced intracellular ROS level, whereas non-targeting siRNA had no obvious effect. In addition, the inhibition of ROS level by SalA was in agreement with the effect induced by VLDL receptor siRNA transfection (Fig. 6).

\section{Discussion}

In the present study, we provided evidence for the first time that SalA, a potential drug component from a medicinal plant, attenuated IS/RP-induced endothelial barrier dysfunction and intracellular ROS generation. The endothelial barrier dysfunction was mediated through the induction of VLDL receptor expression by IS/RP-activated p38 MAPK signaling. Moreover, SalA effectively inhibited IS/RP-induced p38 MAPK activation and VLDL receptor expression. Thus, our results provided a novel mechanism that could contribute to understand the protective action of SalA against IS/RP-induced barrier dysfunction in endothelial cells.

The vascular endothelium is a semi-selective diffusion barrier between the blood plasma and interstitial tissue. It has been previously reported that cytoskeleton reorganization, such as disruption of actin filaments and the formation of stress fibers leads to cell contraction and paracellular gap formation, thereby enhancing endothelial permeability $[1,2,18]$. The formation of paracellular gaps in vascular endothelium is regarded as one of the crucial steps for the development of multiple pathological events, such as inflammation and atheromatous plaque lesions [19, 20]. ROS is accepted generally to be multi-faceted regulator in various pathological states. To date, an accumulated evidence connected ROS generation within cells to the regulation of actin cytoskeleton reorganization and cellular activities driven by this process, such as shape changes, though no interaction between any ROS and actin or actinbinding proteins has been directly demonstrated in vivo.

SalA is one of the main active, water-soluble ingredient in S. miltiorrhiza, which is commonly named "Danshen" in China, and has been widely used as a medicinal plant for more than two thousand years [8]. Moreover, S. miltiorrhiza is an important part in compound danshen dripping pills which have a therapeutic potential on cardiac arrest treatment and have been passed phase II human clinical trial of FDA. Nowadays, modern medical research described many beneficial effects of SalA on cardiovascular system [9]. SalA was suggested to be effective in anti-oxidation, anti-apoptosis, and anti-inflammation in numerous pathological stimuli-induced cardiovascular injury [9]. In the present study, SalA attenuated IS/RP-induced endothelial hyperpermeability (Fig. 1A). Specifically, endothelial actin stress fiber formation and paracellular gap formation were effectively 
inhibited by SalA treatment (Fig. 1B, C). It is well known that tight junctions between cells contributes to barrier function. As an important component of tight junctions, ZO-1 serves as a bridge between transmembrane proteins and cytoskeleton proteins, and this interaction is important to the stability and function of endothelial barrier. In our results, IS/RP-induced discontinuous distribution and decreased intensity of ZO-1 staining were largely restored by SalA treatment (Fig. 1B). The generation of ROS triggers actin cytoskeleton reorganization $[21,22]$. Thus we tested the effect of SalA on intracellular ROS level. Notably, our results showed that IS/RP promoted intracellular ROS level, which was markedly inhibited by SalA treatment in a dose-dependent manner (Fig. 2). Taken these results together, SalA maybe functioned as a protective agent in IS/RP-induced endothelial barrier dysfunction.

Previously, it was proposed that the VLDL receptor mediated the delivery of VLDLderived lipids to peripheral tissues, because it bound VLDL with high affinity in cultured cells and was expressed at high concentrations in tissues that take up VLDL-derived lipids, including muscle, heart, fat, and brain. Interestingly, the VLDL receptor was also expressed at high levels on the endothelial surface [23], however no further information existed on the correlation between VLDL receptor and endothelial cell biology, except for cellular lipid metabolism. Studies indicated that VLDL receptor was essential for the inhibition of cell division in microvascular endothelial cells and that loss of VLDL receptor activated retinal vascular endothelial cells and promoted angiogenesis [6, 7], suggesting a essential role of VLDL receptor in regulation of endothelial activity. It is likely a general phenomenon that VLDL receptor expression is promoted by hypoxia, as hypoxia has previously been shown to increase Vldlr mRNA expression in Muller cells [24] and human monocytes [25]. In agreement with those findings, our study showed that IS/RP induced VLDL receptor expression at both mRNA and protein levels (Fig. 3A, B). Importantly, inactivation of p38 MAPK suppressed the expression of VLDL receptor (Fig. 3C), suggesting that p38 MAPK signaling play a role in the IS/RP-induced VLDL receptor expression. Notably, VLDL receptor (VLDL-R) knockdown experiments further supported a causal relationship between IS/RP-mediated induction of VLDL receptor expression and endothelial barrier dysfunction and intracellular ROS generation (Fig. 5 and Fig. 6).

Recently, mechanisms elucidating the role of SalA in regulation of biological processes in endothelial cells, vascular smooth muscle cells, and cardiomyocytes have been investigated [26-28]. It has been described that SalA might be a cardiovascular protective agent during IS/RP injury, mainly through its anti-apoptosis activity via induction of $\mathrm{Bcl}-2$ and inhibition of Bax $[29,30]$. Further, anti-inflammatory property of SalA was suggested as well which was correlated with the inhibition of granulocyte adherence [31]. Our results provided evidence that SalA markedly inhibited p38 MAPK activation and VLDL receptor expression (Fig. 4A) thus suggesting a novel mechanism to demonstrate the beneficial effect of SalA on vascular health.

In conclusion, our results demonstrated, for the first time, that SalA repressed IS/RPinduced endothelial barrier dysfunction and intracellular ROS generation in HUVECs via inhibited expression of VLDL receptor that represented a novel and crucial mediator of the barrier dysfunction in endothelial cells. Elucidation of the molecular mechanisms by which SalA preserves the barrier function of endothelial cells may have significance for therapy to reduce vascular IS/RP injury.

\section{Acknowledgements}

This work was supported by Shanghai Natural Science Foundation (12411961800) and outstanding youth medical talents of health system in Pudong New Area of Shanghai granted to Dan Yang (PWRq2013-10). 
Yang et al.: SalA Protects IS/RP-Induced Endothelial Barrier Dysfunction

\section{References}

1 Brett J, Gerlach H, Nawroth P, Steinberg S, Godman G, Stern D: Tumor necrosis factor/cachectin increases permeability of endothelial cell monolayers by a mechanism involving regulatory g proteins. J Exp Med 1989;169:1977-1991.

-2 Wojciak-Stothard B, Entwistle A, Garg R, Ridley AJ: Regulation of tnf-alpha-induced reorganization of the actin cytoskeleton and cell-cell junctions by rho, rac, and cdc42 in human endothelial cells. J Cell Physiol 1998;176:150-165.

-3 Bogatcheva NV, Dudek SM, Garcia JG, Verin AD: Mitogen-activated protein kinases in endothelial pathophysiology. J Investig Med 2003;51:341-352.

-4 Tacken PJ, Hofker MH, Havekes LM, van Dijk KW: Living up to a name: The role of the vldl receptor in lipid metabolism. Curr Opin Lipidol 2001;12:275-279.

-5 Takahashi S, Sakai J, Fujino T, Miyamori I, Yamamoto TT: The very low density lipoprotein (vldl) receptor-a peripheral lipoprotein receptor for remnant lipoproteins into fatty acid active tissues. Mol Cell Biochem 2003;248:121-127.

6 Oganesian A, Armstrong LC, Migliorini MM, Strickland DK, Bornstein P: Thrombospondins use the vldl receptor and a nonapoptotic pathway to inhibit cell division in microvascular endothelial cells. Mol Biol Cell 2008;19:563-571.

7 Jiang A, Hu W, Meng H, Gao H, Qiao X: Loss of vldl receptor activates retinal vascular endothelial cells and promotes angiogenesis. Invest Ophthalmol Vis Sci 2009;50:844-850.

-8 Wang L, Zhou GB, Liu P, Song JH, Liang Y, Yan XJ, Xu F, Wang BS, Mao JH, Shen ZX, Chen SJ, Chen Z: Dissection of mechanisms of chinese medicinal formula realgar-indigo naturalis as an effective treatment for promyelocytic leukemia. Proc Natl Acad Sci U S A 2008;105:4826-4831.

-9 Ho JH, Hong CY: Salvianolic acids: Small compounds with multiple mechanisms for cardiovascular protection. J Biomed Sci 2011;18:30.

-10 Hempel SL, Haycraft DL, Hoak JC, Spector AA: Reduced prostacyclin formation after reoxygenation of anoxic endothelium. Am J Physiol 1990;259:C738-745.

11 Yang D, Guo S, Zhang T, Li H: Hypothermia attenuates ischemia/reperfusion-induced endothelial cell apoptosis via alterations in apoptotic pathways and jnk signaling. FEBS Lett 2009;583:2500-2506.

12 Yang D, Xie P, Guo S, Li H: Induction of mapk phosphatase-1 by hypothermia inhibits tnf-alpha-induced endothelial barrier dysfunction and apoptosis. Cardiovasc Res 2010;85:520-529.

13 Yang D, Xie P, Liu Z: Ischemia/reperfusion-induced mkp-3 impairs endothelial no formation via inactivation of erk1/2 pathway. PLoS One 2012;7:e42076.

14 Gomez-Mendikute A, Cajaraville MP: Comparative effects of cadmium, copper, paraquat and benzo[a] pyrene on the actin cytoskeleton and production of reactive oxygen species (ros) in mussel haemocytes. Toxicol In Vitro 2003;17:539-546.

15 Breitenbach M, Laun P, Gimona M: The actin cytoskeleton, ras-camp signaling and mitochondrial ros in yeast apoptosis. Trends Cell Biol 2005;15:637-639.

-16 Wallace BK, Jelks KA, O'Donnell ME: Ischemia-induced stimulation of cerebral microvascular endothelial cell na-k-cl cotransport involves p38 and jnk map kinases. Am J Physiol Cell Physiol 2012;302:C505-517.

17 Gao F, Yue TL, Shi DW, Christopher TA, Lopez BL, Ohlstein EH, Barone FC, Ma XL: P38 mapk inhibition reduces myocardial reperfusion injury via inhibition of endothelial adhesion molecule expression and blockade of pmn accumulation. Cardiovasc Res 2002;53:414-422.

18 Lum H, Malik AB: Mechanisms of increased endothelial permeability. Can J Physiol Pharmacol 1996;74:787-800.

19 Raines EW, Ross R: Biology of atherosclerotic plaque formation: Possible role of growth factors in lesion development and the potential impact of soy. J Nutr 1995;125:624S-630S.

20 Baines CP, Molkentin JD: Stress signaling pathways that modulate cardiac myocyte apoptosis. J Mol Cell Cardiol 2005;38:47-62.

-21 Choong G, Liu Y, Xiao W, Templeton DM: Cadmium-induced glutathionylation of actin occurs through a rosindependent mechanism: Implications for cytoskeletal integrity. Toxicol Appl Pharmacol 2013;272:423430. 
22 Lv P, Miao SB, Shu YN, Dong LH, Liu G, Xie XL, Gao M, Wang YC, Yin YJ, Wang XJ, Han M: Phosphorylation of smooth muscle 22alpha facilitates angiotensin ii-induced ros production via activation of the pkcdeltap47phox axis through release of pkcdelta and actin dynamics and is associated with hypertrophy and hyperplasia of vascular smooth muscle cells in vitro and in vivo. Circ Res 2012;111:697-707.

23 Wyne KL, Pathak K, Seabra MC, Hobbs HH: Expression of the vldl receptor in endothelial cells. Arterioscler Thromb Vasc Biol 1996;16:407-415.

-24 Loewen N, Chen J, Dudley VJ, Sarthy VP, Mathura JR Jr: Genomic response of hypoxic muller cells involves the very low density lipoprotein receptor as part of an angiogenic network. Exp Eye Res 2009;88:928-937.

25 Nakazato K, Ishibashi T, Nagata K, Seino Y, Wada Y, Sakamoto T, Matsuoka R, Teramoto T, Sekimata M, Homma Y, Maruyama Y: Expression of very low density lipoprotein receptor mrna in circulating human monocytes: Its up-regulation by hypoxia. Atherosclerosis 2001;155:439-444.

26 Yang XY, Qiang GF, Zhang L, Zhu XM, Wang SB, Sun L, Yang HG, Du GH: Salvianolic acid a protects against vascular endothelial dysfunction in high-fat diet fed and streptozotocin-induced diabetic rats. J Asian Nat Prod Res 2011;13:884-894.

27 Zhang YQ Tang Y, Wu AL, Zhu HB: Salvianolic acid a displays cardioprotective effects in in vitro models of heart hypoxia/reoxygenation injury. J Asian Nat Prod Res 2010;12:899-915.

28 Oh KS, Oh BK, Mun J, Seo HW, Lee BH: Salvianolic acid a suppress lipopolysaccharide-induced nf-kappab signaling pathway by targeting ikkbeta. Int Immunopharmacol 2011;11:1901-1906.

29 Pan H, Li D, Fang F, Chen D, Qi L, Zhang R, Xu T, Sun H: Salvianolic acid a demonstrates cardioprotective effects in rat hearts and cardiomyocytes after ischemia/reperfusion injury. J Cardiovasc Pharmacol 2011;58:535-542.

-30 Fan H, Yang L, Fu F, Xu H, Meng Q Zhu H, Teng L, Yang M, Zhang L, Zhang Z, Liu K: Cardioprotective effects of salvianolic acid a on myocardial ischemia-reperfusion injury in vivo and in vitro. Evid Based Complement Alternat Med 2012;2012:508938.

31 Jiang M, Wang XY, Zhou WY, Li J, Wang J, Guo LP: Cerebral protection of salvianolic acid a by the inhibition of granulocyte adherence. Am J Chin Med 2011;39:111-120. 